\title{
Consistency of clinical diagnosis in a community-based longitudinal study of dementia and Alzheimer's disease
}

\author{
P.W. Schofield, FRACP; M. Tang, PhD; K. Marder, MD, MPH; K. Bell, MD; \\ G. Dooneief, MD, MPH; R. Lantigua, MD; D. Wilder, PhD; B. Gurland, MD; \\ Y. Stern, PhD; and R. Mayeux, MD, MSE
}

\begin{abstract}
Article abstract-We evaluated the consistency of the diagnosis of dementia in a multicultural, longitudinal community-based study of cognitive impairment and dementia. We diagnosed dementia using a fixed neuropsychological paradigm; the diagnosis also required historical evidence of functional impairment. In a sample of 656 subjects with at least one annual follow-up examination, dementia was confirmed at 1 year in $89 \%$ of the 304 subjects initially demented, and in $90 \%$ of the 136 subjects with the initial diagnosis of probable Alzheimer's disease (AD). The 34 initially demented subjects who failed to meet criteria for dementia at follow-up included 13 with an initial diagnosis of probable $\mathrm{AD}$. All 34 still had evidence of cognitive impairment; this group was more likely to have a history of pulmonary disease, multiple medication use, or chronic alcohol use than other demented patients. Consistency of dementia diagnosis did not vary according to educational attainment or ethnic background. The use of a neuropsychological paradigm such as ours in large longitudinal studies of dementia may minimize interobserver diagnostic variability or diagnostic drift over time while contributing the benefits of a comprehensive cognitive evaluation to the diagnostic process.
\end{abstract}

NEUROLOGY 1995;45:2159-2164

Community-based epidemiologic studies have not addressed the consistency of the diagnosis of dementia, and specifically of Alzheimer's disease $(\mathrm{AD})$, over time. We analyzed data from a longitudinal study of aging and dementia in North Manhattan to look at this issue. We evaluated whether variation in cultural backgrounds and educational experience in our community influenced the consistency of our diagnoses. Of particular interest were initially demented subjects who failed to meet dementia criteria at follow-up. We sought to identify factors to account for this diagnostic change.

Methods. Setting and subjects. Data were obtained from a total of 1,764 subjects who were part of a registry for degenerative disease in the elderly residing in the Washington Heights and Inwood communities of New York City during the period of April 1, 1988, to December 31,
1990. Briefly, the registry used information from all available medical sources-regional hospitals (including inpatient and outpatient services), private practitioners in the community, federal and state health agencies, health maintenance organizations, and senior centersto identify individuals with possible cognitive impairment, who then underwent a cognitive screening evaluation. A proportion of those subjects screened underwent a subsequent comprehensive evaluation, described below. Each reporting site also identified healthy elderly who also took the screening examination and served as controls in some investigations. The refusal rate for both cases and controls was less than $20 \%$ using this method.

Data collection. Information regarding current age, date of birth, location of birth, residence since birth, occupation, and duration of formal education was obtained in the initial interview or record review. For ethnic group assignment, a modification of the United States Census Bureau $^{1}$ format was used. Subjects who identified themselves as Hispanic were classified as such. Non-Hispan-

From the Gertrude H. Sergievsky Center (Drs. Schofield, Tang, Marder, Bell, Dooneief, Stern, and Mayeux); the Center for Geriatrics and Gerontology (Drs. Lantigua, Wilder, and Gurland); the Division of Epidemiology (Dr. Mayeux), Columbia University School of Public Health; the Center for Alzheimer's Disease Research in the City of New York (Drs. Lantigua, Wilder, Gurland, Stern, and Mayeux); and the Departments of Neurology (Drs. Schofield, Tang, Marder, Bell, Dooneief, Stern, and Mayeux) and Psychiatry (Drs. Wilder, Gurland, Stern, and Mayeux) and the Division of General Medicine (Dr. Lantigua), College of Physicians and Surgeons, Columbia University, New York, NY.

Supported by federal grants AG07232, AG10963, AG08702, and RR00645, and the Charles S. Robertson Memorial Gift for Alzheimer's Disease Research from the Banbury Fund.

Received October 18, 1994. Accepted in final form April 13, 1995.

Address correspondence and reprint requests to Dr. R. Mayeux, G.H. Sergievsky Center, 630 West 168 th Street, Columbia University, New York, NY 10032. 
ics were further classified as white, black, or other.

Medical evaluation. This was done as part of the comprehensive evaluation, usually in the subject's home. Past and current medical history and medications were documented in a semistructured format. History of chronic alcohol consumption was recorded categorically, ie, yes/no, without quantification or the use of specific probes. If the subject was unable to provide history or appeared to be demented, information was obtained from available relatives or, in some cases, from a home health aide. Performance of activities of daily living was rated using part 1 of the Blessed Dementia Rating Scale ${ }^{2}$ and the Schwab and England rating scale of activities of daily living. ${ }^{3}$ Interviews were conducted either in Spanish or English, according to the subject's wishes. Neurologic and brief general physical examinations were performed on all subjects.

Neuropsychological evaluation. Comprehensive evaluation included a neuropsychological assessment ${ }^{4}$ comprising tests of orientation, verbal and nonverbal memory, attention, construction, language, and abstract reasoning. A fixed paradigm was used to determine if a subject had sufficient cognitive deficit to meet criteria for dementia. ${ }^{4}$

Diagnostic conference. At a consensus conference, all the information derived from the neurologic and neuropsychological assessments, together with any available ancillary information (eg, CT results, laboratory or pathology results), was reviewed by a group of physicians and neuropsychologists, some of whom were directly involved in the assessment of the patient. A modified Clinical Dementia Rating (CDR) score ${ }^{5}$ was assigned to reflect severity of dementia, taking account of all available information.

Evaluations were repeated annually. After each visit, data were collected and forwarded to the consensus conference for review. For follow-ups, information available from the previous visit was reviewed after a provisional consensus diagnosis was achieved. Any previously collected information such as CT results or history of stroke or psychiatric illness was considered in formulating the definitive consensus diagnosis, the basis for these analyses.

Specific diagnostic criteria. Dementia. Diagnostic criteria for dementia were slightly modified from DSM III$R .^{6}$ A fixed paradigm was used to establish the presence of intellectual dysfunction based on neuropsychological test performance, which was compared with a series of cutoff scores developed in a pilot study in the community. ${ }^{4}$ No adjustments were made in either cutoffs or subject scores according to educational attainment. Using this paradigm, we diagnosed dementia if the subject scored below cutoffs in at least two of three memory tests and below cutoffs in at least two other cognitive areas, including abstract thinking, constructional ability, language, and orientation, and had evidence of impaired activities of daily living or social or occupational function. Dementia was not diagnosed if delirium or altered consciousness was present, and may alone have accounted for the cognitive impairment.

Alzheimer's disease. Operational criteria for this diagnosis were modeled after those recommended by the NINCDS-ADRDA Work Group ${ }^{7}$ for possible and probable $\mathrm{AD}$. We also applied the diagnosis of probable or possible $\mathrm{AD}$ with concomitant stroke to patients with stroke if the dementia syndrome began more than 3 months after stroke or if behavioral deficits due to stroke were superimposed on a preexisting dementing syndrome. When judged by the neuropathologic findings at autopsy, the
Table 1. Patient characteristics according to cognitive function at initial evaluation

\begin{tabular}{|cllll|}
\hline & $\begin{array}{l}\text { No cognitive } \\
\text { impairment } \\
\text { (n=123) }\end{array}$ & $\begin{array}{l}\text { Cognitive } \\
\text { impairment } \\
(\mathbf{n = 2 2 9})\end{array}$ & $\begin{array}{l}\text { Demented } \\
(\mathbf{n}=304)\end{array}$ & $\begin{array}{l}\text { Total } \\
(\mathbf{n}=656)\end{array}$ \\
Age (yr)* & $72.8(6.7)$ & $77.0(7.5)$ & $81.7(7.8)$ & $78.4(8.3)$ \\
Gender (\% F) & 78 & 76 & 73 & 75 \\
Ethnicity & & & & \\
\% White & 15 & 14 & 15 & 15 \\
\% Black & 28 & 31 & 42 & 35 \\
\% Hispanic & 57 & 55 & 43 & 50 \\
Education & $9.3(4.2)$ & $7.0(4.2)$ & $6.8(4.2)$ & $7.4(4.3)$ \\
(yr)* & & & & \\
* Mean, with standard deviation in parentheses. & \\
\hline
\end{tabular}

accuracy of a clinical diagnosis of $\mathrm{AD}$ in subjects participating in the registry exceeds $90 \%$.

Cerebrovascular dementia. The diagnosis of "dementia related to focal effects of stroke" was made when stroke appeared to be the only cause for dementia.

Cognitive impairment. This diagnosis was applied to subjects who did not meet criteria for dementia but who had more than one neuropsychological test score below previously determined cutoffs, and to subjects who satisfied neuropsychological criteria for dementia but who lacked evidence of functional impairment.

Data analysis. To facilitate analyses, subjects were separated into four groups. "Nondemented" (NoDem) subjects were not demented at either initial or follow-up evaluations. "Incident dementia" (IDem) subjects were not demented initially, but were demented at follow-up 1 year later. "Stable dementia" (SDem) subjects were demented at both evaluations, and "unstable dementia" (UDem) subjects were demented initially but not at follow-up. Three different analytic approaches were taken. First, between-group comparisons of demographic and medical variables, and the frequencies of specific causes for dementia, were made using one-way ANOVA $^{8}$ or chi-square tests. ${ }^{9}$ Second, record review of subjects with UDem was undertaken seeking any obvious reason for the cognitive improvement between evaluations. Finally, logistic regression analyses ${ }^{10}$ with dementia group (SDem or UDem) as the outcome variable were performed to evaluate whether specific demographic or medical factors were associated with diagnostic inconsistency.

Results. Of the 1,764 subjects screened, comprehensive evaluation was performed on a sample of 1,058 composed of both screen-positive and screennegative subjects. We report here on the 656 subjects who had at least one subsequent annual follow-up evaluation. The 402 without follow-up, excluded from this analysis, included $22 \%$ who died before the next follow-up, $29 \%$ with evaluations pending, $20 \%$ who had moved or could not be located, $18 \%$ who were ill or were temporarily unavailable, and $11 \%$ who refused follow-up.

Subjects included 493 women and 163 men. At baseline, 304 were demented, 229 were cognitively impaired, and 123 had no cognitive impairment (table 1). Cognitively normal subjects were younger and had more years of education than did the other 
Table 2. Patient characteristics at initial evaluation according to dementia category based on initial and follow-up evaluations

\begin{tabular}{|c|c|c|c|c|}
\hline & $\begin{array}{l}\text { Nondemented } \\
(\mathbf{n}=\mathbf{3 5 2})\end{array}$ & $\begin{array}{l}\text { Incident* } \\
\text { dementia } \\
(\mathbf{n}=63)\end{array}$ & $\begin{array}{l}\text { Unstable }{ }^{\prime} \\
\text { dementia } \\
(\mathbf{n}=\mathbf{3 4})\end{array}$ & $\begin{array}{l}\text { Stable } \neq \\
\text { dementia } \\
(\mathbf{n}=\mathbf{2 7 0})\end{array}$ \\
\hline Age $(y r) ß$ & $74.6(7.1)$ & $80.0(7.8)$ & $78.2(7.1)$ & $82.2(7.8)$ \\
\hline Gender $(\% \mathrm{~F})$ & 79 & 65 & 71 & 74 \\
\hline \multicolumn{5}{|l|}{ Ethnicity } \\
\hline \% White & 14 & 16 & 12 & 16 \\
\hline \% Black & 31 & 26 & 48 & 41 \\
\hline$\%$ Hispanic & 55 & 57 & 39 & 44 \\
\hline Education $(\mathrm{yr}) \S$ & $8.1(4.1)$ & $6.5(5.0)$ & $6.6(3.7)$ & $6.9(4.3)$ \\
\hline Total recall\$ & $35.3(8.8)$ & $28.2(6.1)$ & $20.9(4.5)$ & $15.9(7.5)$ \\
\hline No. medications\$ & $2.3(1.7)$ & $2.6(1.8)$ & $3.1(1.9)$ & $2.1(1.5)$ \\
\hline $\begin{array}{l}\text { History of } \\
\text { chronic alcohol } \\
\text { use }(\%)\end{array}$ & 7 & 7 & 15 & 3 \\
\hline $\begin{array}{l}\text { History of } \\
\text { chronic } \\
\text { pulmonary } \\
\text { disease (\%) }\end{array}$ & 12 & 9 & 24 & 8 \\
\hline $\begin{array}{l}\text { History of } \\
\text { hypertension }(\%)\end{array}$ & 55 & 57 & 84 & 56 \\
\hline \multicolumn{5}{|c|}{$\begin{array}{l}\text { * Subjects first diagnosed demented at follow-up. } \\
\dagger \text { Subjects diagnosed demented at initial evaluation, but not at fol- } \\
\text { low-up. } \\
\ddagger \text { Subjects diagnosed demented at both evaluations. } \\
\S \text { Mean, with standard deviation in parentheses. } \\
\text { I Total recall of the Buschke Selective Reminding Test. }\end{array}$} \\
\hline
\end{tabular}

two categories (Tukey honestly significant difference [HSD], <0.05). Cognitively impaired subjects were younger than those with dementia (Tukey HSD, <0.05). At 1-year follow-up, there were 63 incident cases of dementia, of whom four had previously been cognitively normal and 59 were previously considered cognitively impaired. In 34 of the 304 subjects demented at initial evaluation, dementia was not confirmed at 1-year follow-up because in every case the neuropsychological criteria for dementia were no longer met. Although not demented at follow-up, all 34 subjects still had evidence of some cognitive impairment. Of the 304 initially demented, 238 had a diagnosis of $\mathrm{AD}$ (ie, possible $\mathrm{AD}$ or probable $\mathrm{AD}$ ). At follow-up, 215 of these had diagnoses of $A D$, one had a diagnosis of "dementia cause unknown," and 23 no longer met criteria for dementia. When only cases with an initial diagnosis of probable $\mathrm{AD}$ were considered $(\mathrm{n}=136)$, follow-up diagnoses included probable $\mathrm{AD}$ in 111, possible $\mathrm{AD}$ in 12 , and 13 no longer met criteria for dementia.

The 656 subjects comprised 289 NoDem, 63 IDem, 34 UDem, and 270 SDem subjects. Table 2 lists subject characteristics within these categories. NoDem subjects were significantly younger than subjects in the other three groups, and SDem subjects were in addition older than IDem and UDem subjects (Tukey HSD, <0.05). NoDem subjects had more years of education than did the remaining three groups (Tukey HSD, <0.05). UDem subjects were more likely to have a history of hypertension
Table 3. Etiology of dementia at initial evaluation

\begin{tabular}{|lcc|}
\hline & $\begin{array}{l}\text { Stable } \\
\text { dementia* } \\
(\mathbf{n = 2 7 0 )}\end{array}$ & $\begin{array}{l}\text { Unstable } \\
\text { dementia } \\
(\mathbf{n}=\mathbf{3 4})\end{array}$ \\
$\begin{array}{l}\text { Probable AD } \\
\text { Possible AD }\end{array}$ & $46 \%(123)$ & $38 \%(13)$ \\
Vascular-related & $34 \%(92)$ & $29 \%(10)$ \\
dementia & $7 \%(20)$ & $6 \%(2)$ \\
Secondary dementia & $1 \%(2)$ & $3 \%(1)$ \\
Other dementia & $6 \%(17)$ & $17 \%(6)$ \\
Dementia cause & $6 \%(16)$ & $6 \%(2)$ \\
unknown & & \\
$*$ Demented at both initial and follow-up evaluations. \\
$\dagger$ Demented at initial evaluation only. \\
AD Alzheimer's disease.
\end{tabular}

(chi-square Pearson, 0.013) and took more medications (Tukey HSD, <0.05) than did subjects from the other diagnostic categories. UDem subjects took on average the same number of medications at both evaluations. When compared with IDem and SDem subjects, UDem subjects were more likely to have a history of chronic pulmonary disease (chisquare, 0.02) and chronic alcohol use (chi-square, 0.02 ). Within the population as a whole, the single memory item that correlated most strongly with CDR was the total recall of the Buschke Selective Reminding Test. ${ }^{11}$ The mean performance on this test at initial evaluation was significantly different for all four groups (Tukey HSD, <0.05).

Etiology of the dementia in the UDem and SDem groups at initial evaluation is outlined in table 3. A greater proportion of UDem subjects than of SDem subjects had diagnoses other than $A D$, although this was not a statistically significant difference.

Chart review of UDem subjects was undertaken seeking additional history that might explain why cognitive improvement occurred between successive evaluations. In three subjects, stroke symptoms occurred within 5 months before the first evaluation. In four other subjects, a direct plausible explanation for the improvement was readily suggested. These four cases are outlined below.

Patient 1. A 65-year-old woman with chronic lung disease recently complicated by bilateral pneumothorax that required 24-hour oxygen was initially evaluated as demented, with a CDR score of 1 . One year later she performed somewhat better, and was rated CDR 0.5 .

Patient 2. A 91-year-old woman was diagnosed with probable AD (PAD), with a CDR score of 2 . She had recently been admitted to the hospital after a rapid decline, and CT had excluded stroke. When seen 1 year later, she was much improved, with a CDR of 0 .

Patient 3. A 74-year-old man initially diagnosed with PAD and a CDR of 1 had a CDR of 0 the following year. Subsequently, a history of "drinking alcohol all the time" was obtained. 
Patient 4. An 81-year-old woman with diabetes, hypertension, and peripheral vascular disease with possible right-sided facial weakness was diagnosed as demented, with a CDR of 1 . One year later, her CDR was 0 , and the same examiner this time noted no equivocal lateralized neurologic signs.

In retrospect, lung disease with hypoxemia/hypercapnia, a resolving confusional state, alcohol-related cognitive problems (possibly intoxication), and stroke may have accounted for the poorer performance on testing at the first evaluations. While no longer meeting criteria for dementia at followup evaluation, all four subjects continued to show evidence of cognitive impairment.

Concomitant medical conditions and medications were evaluated as factors that might contribute to the stability of dementia diagnosis. A logistic model was created in which the dichotomous outcome variable was a dementia group, either SDem or UDem. Potential confounders or contributing factors evaluated as independent variables included the following: medications taken at initial evaluation; a history of chronic medical conditions including congestive heart failure, chronic renal disease, chronic pulmonary disease, chronic liver disease, thyroid disease, hypertension, and diabetes; a history of chronic alcohol consumption; or head injury with loss of consciousness. Ethnicity, age, and education as a dichotomous variable (less than 8 or 8 or more years of school) were evaluated in all logistic models. Three different categories of medications were created for inclusion in the logistic model. The first category comprised the number of the medications listed, from which a dichotomous variable (less than three or three or more medications) was created. A second category consisted of the number of medications recognized to more commonly be associated with cognitive impairment, and a dichotomous variable (one or less or two or more medications) was created. Finally, a category of antihypertensives was created. Only one medication category was evaluated in the logistic model at any time. In order to control for the initial severity of cognitive impairment, analyses were restricted to a sample comprising 31 UDem subjects and 126 SDem subjects, all with an initial CDR of 1 . In these analyses, a history of chronic pulmonary disease (odds ratio [OR], 5.9; 95\% confidence interval [CI], 1.6 to 21.7), three or more medications (OR, $3.1 ; 95 \% \mathrm{CI}, 1.3$ to 7.9 ), and a history of chronic alcohol intake (OR, 4.8; 95\% CI, 1.0 to 22.1) were all independently predictive of UDem diagnosis. None of the other medical conditions or ethnicity, age, or education were significant predictors of dementia category, and they were excluded from the model. When we added the total recall from the Buschke Selective Reminding Test as a continuous variable to the previous model to adjust for the baseline severity of memory impairment, better performance was associated with increased likelihood of UDem (OR, 1.1; 95\% CI, 1.0 to 1.2 ), but there was little change in the odds ratios associated with the other significant predictors. The analyses were repeated with the study population further restricted to UDem and SDem patients with the initial diagnosis of PAD and a CDR of 1 . Chronic pulmonary disease (OR, 13.8; 95\% CI, 1.6 to 118.6 ) and a history of taking more than three medications (OR, 9.2; $95 \% \mathrm{CI}, 1.5$ to 58.5 ) were independently associated with increased risk for unstable diagnosis, and a history of hypertension also conferred increased risk (OR, 8.0; 95\% CI, 0.68 to 93.5), although this was not statistically significant. Once again, ethnicity, educational attainment, and age were not significant predictors and were excluded from the model.

Discussion. About $90 \%$ of subjects diagnosed initially as demented, or with probable AD specifically, were again demented at 1-year follow-up. There were roughly one-half as many demented patients whose diagnosis changed as there were patients with IDem. A history of chronic pulmonary disease, taking three or more medications, and chronic alcohol use were all independently associated with an increased likelihood that an initial diagnosis of dementia would not be confirmed at follow-up.

The neuropsychological paradigm and independent assessment of function that we employed provide explicit operational criteria for a diagnosis of dementia. One consequence of defining a precise threshold for dementia diagnosis is that very small changes in neuropsychological performance may lead to diagnostic changes, and the biological meaning of these diagnostic changes may be complex. "Recovery" from dementia should not be unexpected. ${ }^{12,13}$ Improvement in cognitive performance over successive evaluations may even be seen in patients with probable $A D$. In two recent longitudinal studies of patients meeting NINCDS-ADRDA criteria for probable $\mathrm{AD}$, six of $110^{14}$ and five of 54 patients ${ }^{15}$ showed improved cognition at 12 or more months of follow-up.

There are four settings in which a patient with an initial diagnosis of dementia might show apparent improvement on a subsequent evaluation. First, patients with nonprogressive or slowly progressive cognitive impairment whose neuropsychological performance is at or near threshold for dementia diagnosis will be especially likely to change diagnostic category with repeat testing. In such subjects, minor variations in test score could readily swing them back across the diagnostic threshold. The results of our logistic analyses confirm the importance of proximity to threshold as a risk for this change in dementia diagnosis: demented subjects with better scores for "total recall"-ie, closer to cutoffs-were less likely to be demented at follow-up. Second, an intercurrent event (eg, small stroke, infection, etc) occurring just before the first assessment might lead to an impression of improvement, despite the presence of an underlying dementing process such as AD. Subsequent recov- 
ery from the effects of the preceding event might permit better performance at follow-up than at initial examination. Several of the case vignettes outlined above represent possible examples of such a mechanism. Third, marked variability of cognition over shorter time intervals might also lead to an apparent improvement in cognition in some patients despite a declining trend, with first evaluations representing nadir performances and second evaluations representing peak performances. Such a mechanism might account for the association of chronic pulmonary disease and perhaps alcohol consumption with UDem, discussed further below. Finally, resolution or improvement of a reversible condition that was completely responsible for the initial dementia might account for the improvement in some subjects (ie, a true reversible dementia versus the mixed pathology invoked for the second mechanism). Within a population of individuals including a majority with progressive dementia, biological factors causally linked with the four situations outlined above but not associated with the dementia process itself might emerge as factors that predict dementia that is likely to vary.

Chronic pulmonary disease was associated with an increased likelihood that the diagnosis of dementia would not be confirmed at follow-up. Neuropsychological abnormalities with a distinctive pattern of deficits ${ }^{16}$ occur in association with severe chronic obstructive pulmonary disease (COPD), ${ }^{17}$ and such patients may improve after chronic oxygen administration. ${ }^{18}$ of 200 consecutive patients with dementia, COPD was present as a treatable condition in 20; treatment recommendations were made in at least five patients, but it is unclear to what extent these were associated with cognitive improvement. ${ }^{13}$ Chronic obstructive lung disease may cause fluctuations in cognitive impairment reflecting hypoxemia/hypercapnia. ${ }^{17}$ Our patient 4 required home supplemental oxygen.

A history of taking three or more prescription medications daily at baseline was also associated with diagnostic changes from dementia to cognitive impairment. Adverse drug reactions may contribute to the cognitive impairment due to dementing disorders such as $\mathrm{AD}$, and cognition may improve with cessation of the responsible drug. ${ }^{13,19-21}$ In our series, cognitive improvement was not due to a deliberate withdrawal of medications. Since compliance correlates inversely with the number of prescription medications, ${ }^{22,23}$ however, these subjects may have had a more variable intake compared with subjects whose diagnosis remained unchanged.

A history of chronic alcohol use was also associated with increased likelihood of diagnostic change. We did not have reliable quantitative data; however, some subjects' performances on neuropsychological testing may have been affected by recent alcohol consumption.

Hypertension approached significance as a predictor of diagnostic change among subjects with an initial diagnosis of probable AD. There are reports $^{24-27}$ of uncomplicated hypertension associated with cognitive decline, but the decline was less severe than among our patients whose diagnosis changed.

Longitudinal epidemiologic studies of dementia typically require the participation of numerous clinicians who may vary in their application of diagnostic criteria. Over the course of a long study, systematic drift in a clinician's threshold for dementia diagnosis might occur, due for instance to an increasing willingness to accept low educational attainment as the sole explanation for poor performance on cognitive testing. We attempted to minimize the consequences of observer diagnostic variability by specifying invariant neuropsychological criteria for dementia. Low educational attainment did not predict reversal of the diagnosis of dementia at follow-up, suggesting that our cutoffs were not inappropriately stringent: if the cutoff lay within the performance range of normal subjects with low educational attainment, we might have expected them to be overrepresented among the UDem group. On the other hand, because we did not adjust for educational attainment in the diagnostic process, we may have diagnosed individuals with less education earlier in the course of dementing diseases. ${ }^{28}$ Cognitive impairment and dementia represent successive stages on a continuum of cognitive decline, and all our subjects with an initial diagnosis of dementia that changed had persisting cognitive impairment at the repeat evaluation. Prolonged follow-up will clearly be important to determine which of those cognitively impaired subjects have progressive disease.

There are some limitations to this study. Brain imaging was not obtained as a routine part of the subject evaluation. When CTs or MRIs were done, they were obtained by the subjects' own personal physicians. Larson et $\mathrm{al}^{29}$ reviewed the value of CT in the diagnostic evaluation of patients with dementia and concluded that in the absence of a specific clinical suspicion, CT findings rarely lead to a significant change in diagnosis. Nevertheless, the relative lack of laboratory input to the diagnostic process may have led to diagnostic errors in some cases.

In summary, we used a neuropsychological paradigm to diagnose dementia in a longitudinal study of community-dwelling elders at high risk for cognitive decline. Ethnicity and education did not affect the consistency of clinical diagnosis of dementia at 1-year follow-up, whereas a history of chronic pulmonary disease, multiple drug taking, or chronic alcohol use did-findings for which there are obvious or plausible explanations. A neuropsychological paradigm such as ours may protect against inconsistency of dementia diagnosis related to interobserver variability or to systematic drift in the application of diagnostic criteria over the course of a prolonged study. 


\section{References}

1. Census of Population and Housing, 1990: Summary Tape File 1. Technical Documentation Prepared by the Bureau of Census. Washington: The Bureau of Census, 1991.

2. Blessed $G$, Tomlinson BE, Roth $M$. The association between quantitative measures of dementia and of senile changes in the cerebral grey matter of elderly subjects. $\mathrm{Br}$ J Psychol 1968;225:797-811.

3. Schwab JF, England AC. Projection technique for evaluating surgery in Parkinson's disease. In: Gillinghan FS, Donaldson MN, eds. Third symposium on Parkinson's disease. Edinburgh, Scotland: E. \& S. Livingstone, 1969:152-157.

4. Stern Y, Andrews H, Pittman J, et al. Diagnosis of dementia in a heterogeneous population: development of a neuropsychological paradigm-based diagnosis of dementia and quantified correction for the effects of education. Arch Neurol 1992;49:453-460.

5. Burke WJ, Miller JP, Rubin EH, et al. The reliability of the Washington University Clinical Dementia Rating. Arch Neurol 1988;45:31-32.

6. American Psychiatric Association. Diagnostic and statistical manual of mental disorders, revised 3rd ed. Washington, DC: American Psychiatric Press, 1987.

7. McKhann G, Drachman D, Folstein M, Katzman R, Price D, Stadlan EM. Clinical diagnosis of Alzheimer's disease: report of the NINCDS-ADRDA Work Group under the auspices of Department of Health and Human Services Task Force on Alzheimer's Disease. Neurology 1984;34:939-944.

8. Kleinbaum DG, Kupper LL, Muller KE. Applied regression analysis and other multivariable methods. 2nd ed. Belmont, CA: Duxbury Press, 1988.

9. Fleiss Л. Statistical methods for rates and proportions. 2nd ed. New York: John Wiley and Sons, 1981.

10. Breslow NE, Day NE. Statistical methods in cancer research, vol 1 . The analysis of case-control studies. Lyon: International Agency for Research on Cancer, 1980.

11. Buschke H, Fuld PA. Evaluating storage, retention, and retrieval in disordered memory and learning. Neurology 1974;24:1019-1025.

12. Meyer JS, Judd BW, Tawakina T, Rogers RL, Mortel KF. Improved cognition after control of risk factors for multi-infarct dementia. JAMA 1986;256:2203-2209.

13. Larson EB, Reifler BV, Sumi SM, Canfield CG, Chinn NM. Diagnostic evaluation of 200 elderly outpatients with suspected dementia. J Gerontol 1985;40:536-543.

14. Burns A, Jacoby R, Levy R. Progression of cognitive impairment in Alzheimer's disease. J Am Geriatr Soc 1991;39:39-45.
15. Ortof E, Crystal HA. Rate of progression of Alzheimer's disease. J Am Geriatr Soc 1989;37:511-514.

16. Incalzi RA, Gemma A, Marra C, Muzzolon R, Capparella O, Carbonin P. Chronic obstructive pulmonary disease: an original model of cognitive decline. Am Rev Respir Dis 1993;148:418-424.

17. Grant I, Heaton RK, McSweeney AJ, Adams KM, Timms RM. Neuropsychologic findings in hypoxemic chronic obstructive pulmonary disease. Arch Intern Med 1982;142:1470-1476.

18. Heaton RK, Grant I, McSweeney AJ, Adams KM, Petty TL. Psychologic effects of continuous and nocturnal oxygen therapy in hypoxemic chronic obstructive pulmonary disease. Arch Intern Med 1983;143:1941-1947.

19. Larson EB, Kukull WA, Buchner D, Reifler BV. Adverse drug reactions associated with global cognitive impairment in elderly persons. Ann Intern Med 1987;107:169-173.

20. Klein LE, German PS, Levine DM, Feroli ER, Ardery J. Medication problems among outpatients: a study with emphasis on the elderly. Arch Intern Med 1984;144:1185-1188.

21. Task force sponsored by the National Institute on Aging. Senility reconsidered: treatment possibilities for mental impairment in the elderly. JAMA 1980;244:259-263.

22. Darnell JC, Murray MD, Martz BL, Weinberger M. Medication use by ambulatory elderly: an in-home survey. J Am Geriatr Soc 1986;34:1-4.

23. Kendrick R, Bayne JRD. Compliance with prescribed medication by elderly patients. Can Med Assoc J 1982;127:961962.

24. Walstein SR, Manuck SB, Ryan CM, Muldoon MF. Neuropsychological correlates of hypertension: review and methodologic considerations. Psychol Bull 1991;110:451-468.

25. Starr JM, Whalley LJ, Inch S, Shering PA. Blood pressure and cognitive function in healthy old people. J Am Geriatr Soc 1993;41:753-756.

26. Elias MF, Wolf PA, D'Agostino RB, Cobb J, White LR. Untreated blood pressure level is inversely related to cognitive functioning: the Framingham Study. Am J Epidemiol 1993; 138:353-364.

27. Starr JM, Whalley LJ. Senile hypertension and cognitive impairment: an overview. J Hypertens Suppl 1992;10:S31S42.

28. Stern Y, Gurland B, Tatemichi T, Tang M, Wilder D, Mayeux R. Influence of education and occupation on the incidence of Alzheimer's disease. JAMA 1994;271:1004-1010.

29. Larson EB, Reifler BV, Sumi SM, Canfield CG, Chinn NM. Diagnostic tests in the evaluation of dementia: a prospective study of 200 elderly outpatients. Arch Intern Med 1986; 146:1917-1922. 